\title{
SELBERG INTEGRAL OVER LOCAL FIELDS
}

\author{
ZENAN FU AND YONGCHANG ZHU
}

\section{INTRODUCTION}

Selberg introduced his beautiful integral formula in 1944 [Sel] that asserts

$$
\begin{aligned}
S_{n}(a, b, c) & =\int_{0}^{1} \cdots \int_{0}^{1} \prod_{i=1}^{n} t_{i}^{a-1}\left(1-t_{i}\right)^{b-1} \prod_{1 \leq i<j \leq n}\left|t_{i}-t_{j}\right|^{2 c} d t_{1} \cdots d t_{n} \\
& =\prod_{j=0}^{n-1} \frac{\Gamma(a+j c) \Gamma(b+j c) \Gamma(1+(j+1) c)}{\Gamma(a+b+(n+j-1) c) \Gamma(1+c)}
\end{aligned}
$$

where $n$ is a positive integer and $a, b, c$ are complex numbers satisfying re $a>0$, re $b>0$ and re $c>-\min \{1 / n$, re $a /(n-1)$, re $b /(n-1)\}$. We refer to Forrester and Warnaar's exposition [FW] for the history, generalizations and the applications of Selberg integral. Evans [E1] conjectured a finite field analog of Selberg integral formula in 1980. And Anderson [An] proved a major case of it in 1981 and his ideas was used to obtained the complete result [E2]. On the other hand, Aomoto [Ao] proved an analog of Selberg integral for complex field $\mathbb{C}$ in 1987. The purpose of the present paper is to formulate and prove Selberg integral formula for local fields of characteristic zero. To state our results, we first introduce some notations.

Let $F$ be a local field of characteristic zero, $\psi$ be a non-trivial additive character, $d x$ be the self-dual Haar measure with respect to $\psi$, i.e., the Fourier transform defined using $d x$,

$$
\mathcal{F} f(x)=\int_{F} f(y) \psi(x y) d y
$$

is an isometry. The absolute value $|a|_{F}$ of $a \in F$ is defined by the formula $\operatorname{vol}(a U)=|a|_{F} \operatorname{vol}(U)$. For a quasi-character $c$ of $F^{*}$, its real part, denoted by $\operatorname{re} c$, is the unique real number satisfying the condition

$$
|c(x)|=|x|_{F}^{\mathrm{re} c} .
$$

The $\rho$-factor $\rho(c)$ of $c$ is defined as

$$
\rho(c):=\frac{\int f(x) c(x)|x|_{F}^{-1} d x}{\int \mathcal{F} f(x) c^{-1}(x) d x} .
$$

where $f$ is a Schwartz function on $F$, the both integrals converge when $0<$ re $c<1$ and they are understood as analytic continuation for general $c$, see [Tate]. We also use the gamma function $\Gamma(c)$ as in [GGPS], it is related to $\rho(c)$ by $\Gamma(c)=c(-1) \rho(c)$. We denote $\chi_{0}(x)=|x|_{F}$.

The choice of $\psi$ induces an additive character $\psi_{E}$ on any finite extension $E$ by $\psi_{E}(x)=\psi(\operatorname{Tr} x)$ and therefore the self-dual Haar measure $d_{E}$ on $E$. Then for any quasi-character $c$ of $E^{*}$, we can similarly define the $\rho_{E}(c)$ and $\Gamma_{E}(c)$. The absolute value of $E$ is denoted by $|x|_{E}$, and we have $|x|_{E}=|\mathrm{N}(x)|_{F}$. Note that for a quasi-character $c$ of $F^{*}$, its composition $\mathrm{N} \circ c$ with the norm map has the property $\operatorname{re}(\mathrm{N} \circ c)=\operatorname{re} c$.

Date: October 4, 2018. 
Let $M_{n}$ be the space $\{f \in F[x] \mid f$ is monic of degree $n\}$ for $n \in \mathbb{Z}_{>0}$. Equal $M_{n}$ with $F^{n}$ via the map

$$
\eta: M_{n} \rightarrow F^{n}, f(x)=x^{n}+b_{n-1} x^{n-1}+\cdots+b_{0} \mapsto\left(b_{n-1}, \cdots, b_{0}\right) .
$$

Let $d f$ be the measure inherits from the product Haar measure of $F^{n}$. Recall the discriminant $\Delta(f)$ of $f \in M_{n}$ is defined as

$$
\Delta(f)=(-1)^{\frac{1}{2} n(n-1)} \prod_{i \neq j}\left(\alpha_{i}-\alpha_{j}\right)=\prod_{1 \leq i<j \leq n}\left(\alpha_{i}-\alpha_{j}\right)^{2},
$$

where $\alpha_{i}(i=1, \ldots, n)$ are the roots of $f$. Empty product is considered to be equal to 1 . For an irreducible polynomial $h(x)$ over $F$, we denote the field $F[x] /(h(x))$ by $F_{h}$ and if $\chi$ is a quasicharacter of $F^{*}$, we denote $\Gamma_{h}(\chi)$ the Gamma function $\Gamma_{F_{h}}(\chi \circ \mathrm{N})$.

For quasi-characters $\alpha, \beta, \gamma$ of $F^{*}$ in the region $R_{n}$ given by

$$
\begin{aligned}
& \text { re } \alpha, \text { re } \beta, \text { re } \gamma>0 ; \\
& \operatorname{re} \alpha+\operatorname{re} \beta+2(n-1) \operatorname{re} \gamma<1,
\end{aligned}
$$

we define the Selberg integral over $F$ as

$$
S_{n}(\alpha, \beta, \gamma)=\alpha^{n} \gamma^{\frac{n(n-1)}{2}}(-1) \int_{f \in M_{n}} \alpha \chi_{0}^{-1}(f(0)) \beta \chi_{0}^{-1}(f(1)) \gamma \chi_{0}^{-\frac{1}{2}}(\Delta(f)) \prod_{i=1}^{l} \frac{\Gamma_{h_{i}}(\gamma)}{\Gamma(\gamma)^{\operatorname{deg} h_{i}}} d f
$$

where we write $f(x)=\prod_{i=1}^{l} h_{i}(x)$ with $h_{i}$ monic irreducible polynomial over $F$. Note that polynomials with zero discriminant have measure zero, so we may assume $f$ has no repeated roots.

We have the following theorem that generalizes Selberg integral formula (1.1),

Theorem 1.1. The integral $S_{n}(\alpha, \beta, \gamma)$ converges for $(\alpha, \beta, \gamma) \in R_{n}$ and

$$
S_{n}(\alpha, \beta, \gamma)=\prod_{j=0}^{n-1} \frac{\Gamma\left(\alpha \gamma^{j}\right) \Gamma\left(\beta \gamma^{j}\right) \Gamma\left(\gamma^{j+1}\right)}{\Gamma\left(\alpha \beta \gamma^{n+j-1}\right) \Gamma(\gamma)}
$$

In the case $F$ is a finite field, we consider the Gauss sums for $F$ as an analog of the Gamma factors, then the factor $\prod_{i=1}^{l} \frac{\Gamma_{h_{i}}(\gamma)}{\Gamma(\gamma)^{\operatorname{deg} h_{i}}}$ is equal to 1 by the Hasse-Davenport relation, so this term does not appear in the finite field generalization of Selberg integral in [E1] [An] (see also [AAR]). For the case $F=\mathbb{C}$, since all $F_{h}=\mathbb{C}$, the above factor is also equal to $1,(1.8)$ reduces to Aomoto's generalization of Selberg integral [Ao], see the end of Section 3 for more detail. While Aomoto [Ao] considers (1.7) for $F=\mathbb{C}$ as a pairing in certain twisted de Rham cohomology and homology, our integral is just the ordinary integral. Our domain of convergence (1.6) for the case $F=\mathbb{C}$ is contained in Aomoto's defining domain for (1.7). Note that only the unramified quasi-characters $\alpha, \beta, \gamma$ are considered in [Ao]. We also remark that for general $F, f \mapsto \prod_{i=1}^{l} \frac{\Gamma_{h_{i}}(\gamma)}{\Gamma(\gamma)^{\operatorname{deg} h_{i}}}$ is a locally constant function on the region $M_{n}-\{f \mid \Delta(f)=0\}$, which is a disjoint unions of open sets of $f$ 's with $F[x] /(f(x))$ isomorphic to a direct product of extensions of $F$ of fixed types.

We like to comment that (1.8) for the case $F=\mathbb{R}$ is not equivalent to the original Selberg integral (1.1), which should be understood as (1.8) for $\left(\mathbb{R}_{\geq 0},+, \cdot\right)$.

We prove Theorem 1.1 by evaluating a double integral in two different ways, which give a recursive formula relating $S_{n}$ to $S_{n-1}$. This method is due to Anderson [An] [An2].

This paper is organized as follows. In Section 2, we prove an extension of beta integral and two corollaries that are used in the proof of Theorem 1.1. In Section 3, we prove our main theorem and compare our formula in the case $F=\mathbb{C}$ with Aomoto's. 


\section{Generalized Beta Integrals.}

Lemma 2.1. Let $V$ be a finite dimensional vector space over $F, d x$ be a Haar measure on $V$ as an additive group. If $N: V \rightarrow \mathbb{R}_{\geq 0}$ is a $F$-norm, then for re $s<-\operatorname{dim}_{F} V$,

$$
\int_{N(x) \geq r} N(x)^{s} d x
$$

converges for any $r>0$.

Proof. The cases $F=\mathbb{R}, \mathbb{C}$ are standard exercises in calculus. We assume $F$ non-Archimedean. Let $n=\operatorname{dim}_{F} V$. Since all the norms on $V$ are equivalent, we may assume that $V$ is a field extension of $F$ and $N(x)=|x|_{V}^{\frac{1}{n}}$. The result follows from the fact that $\int_{|x|>r}|x|_{V}^{\frac{s}{n}} d x$ converges when $\frac{1}{n}$ re $s<-1$.

It is known that $\rho(c)$ for re $c>0$ can be written as an integral

$$
\rho(c)=c(-1) \int_{F} \psi(x) c(x)|x|_{F}^{-1} d x:=c(-1) \lim _{r \rightarrow \infty} \int_{|x| \leq r} \psi(x) c(x)|x|_{F}^{-1} d x .
$$

Equivalently, the Gamma function of $c$ can be written as

$$
\Gamma(c)=c(-1) \rho(c)=\int_{F} \psi(x) c(x)|x|_{F}^{-1} d x,
$$

see [GGPS]. This formula is used to prove the beta integral formula [GGPS]:

$$
\int_{F} c_{1}(x)|x|^{-1} c_{2}(1-x)|1-x|^{-1} d x=\frac{\Gamma\left(c_{1}\right) \Gamma\left(c_{2}\right)}{\Gamma\left(c_{1} c_{2}\right)} .
$$

where the convergence region is re $c_{1}>0$, re $c_{2}>0$, re $c_{1}+\operatorname{re} c_{2}<1$. See [Tai] page 61 for a detailed proof. We will need the following generalization of (2.4).

Lemma 2.2. Let $E_{1}, \ldots, E_{k}$ be finite extensions of $F$ of degrees $d_{1}, \ldots, d_{k}$ with $d=d_{1}+\cdots+d_{k} \geq 2$, $\operatorname{Tr}_{i}: E_{i} \rightarrow F$ be the trace map, $\phi: E_{1} \times \cdots \times E_{k} \rightarrow F$ be the $F$-linear map given by

$$
\phi\left(x_{1}, \ldots, x_{k}\right)=\sum_{i=1}^{k} \operatorname{Tr}_{i}\left(x_{i}\right) .
$$

Let $S=\phi^{-1}(1)$ and ds be the measure on $S$ uniquely determined by the conditions that it is invariant under translations by $\phi^{-1}(0)$ and that the map

$$
F^{*} \times S \rightarrow E_{1} \times \cdots \times E_{k},\left(a,\left(x_{1}, \ldots, x_{k}\right)\right) \mapsto\left(a x_{1}, \ldots, a x_{k}\right)
$$

changes the measure $|a|^{d-1} d a d s$ to $d y_{1} \cdots d y_{k}$ (where da, dy are self-dual measures defined in Section 1). If $c_{1}, \ldots, c_{k}$ are quasi-characters on $E_{1}^{*}, \ldots, E_{k}^{*}$ with re $c_{i}>0$ for all $i$ and

$$
d_{1} \text { re } c_{1}+\cdots+d_{k} \text { re } c_{k}<1 \text {. }
$$

then the integral

$$
\int_{S} c_{1}\left(x_{1}\right)\left|x_{1}\right|_{E_{1}}^{-1} \cdots c_{k}\left(x_{k}\right)\left|x_{k}\right|_{E_{k}}^{-1} d s
$$

converges and is equal to

$$
\frac{\prod_{i=1}^{k} \Gamma_{E_{i}}\left(c_{i}\right)}{\Gamma(c)}
$$


where $c=\left(\left.c_{1}\right|_{F^{*}}\right) \cdots\left(\left.c_{k}\right|_{F^{*}}\right)$.

Proof. We prove first the convergence by induction. We may assume all $c_{i}$ are $\mathbb{R}_{>0}$ valued, so $c_{i}(x)=|x|_{E_{i}}^{r_{i}}, r_{i} \in \mathbb{R}$. If $k=1$, then $d_{1}>1$, let $v \in E_{1}$ satisfy $\phi(v)=1$, the integral is

$$
\int_{S} c_{1}\left(x_{1}\right)\left|x_{1}\right|_{E_{1}}^{-1} d s=\int_{\phi^{-1}(0)}|y+v|_{E_{1}}^{r_{1}-1} d y
$$

where we change the variable $x_{1} \rightarrow y+v$ and $d y$ is the Haar measure on $\phi^{-1}(0)$ induced from $d s$ on $S$. Since $y+v$ is never 0 , the integrand has no finite singular point, it is enough to prove

$$
\int_{y: y \in \phi^{-1}(0),|y| \geq r}|y+v|_{E_{1}}^{r_{1}-1} d y<\infty
$$

for any $r>0$. Note

$$
|y+v|_{E_{1}}^{r_{1}-1}<C|y|_{E_{1}}^{\text {re } c_{1}-1}
$$

for $y$ with $|y|_{E_{1}}>r$ ( $C$ is a constant depending on $r$ ). So it is enough to prove

$$
\int_{|y|_{E_{1}} \geq r}|y|_{E_{1}}^{r_{1}-1}<\infty
$$

Notice that $y \mapsto|y|_{E_{1}}^{\frac{1}{d_{1}}}$ is an $F$-norm. The result follows from Lemma 2.1 for $V=\phi^{-1}(0)$.

The case $k=2$ and $d_{1}=d_{2}=1$ is (2.4). The case $k=2$ and $d_{1}+d_{2}>2$ can be proved using the induction on $d_{1}+d_{2}$. For $k \geq 3$, we have

$$
\begin{aligned}
& \int_{S} c_{1}\left(x_{1}\right)\left|x_{1}\right|_{E_{1}}^{-1} \cdots c_{k}\left(x_{k}\right)\left|x_{k}\right|_{E_{k}}^{-1} d x \\
= & \int_{\operatorname{Tr} x_{1}+\operatorname{Tr} x_{2}=1} c_{1}\left(x_{1}\right)\left|x_{1}\right|_{E_{1}}^{-1} c_{2}\left(x_{2}\right)\left|x_{2}\right|_{E_{2}}^{-1} d\left(x_{1}, x_{2}\right) \\
& \cdot \int_{a+\operatorname{Tr} x_{3}+\cdots+\operatorname{Tr} x_{k}=1}|a|^{d_{1} r_{1}+d_{2} r_{2}-1} c_{3}\left(x_{3}\right)\left|x_{3}\right|_{E_{3}}^{-1} \cdots c_{k}\left(x_{k}\right)\left|x_{k}\right|_{E_{k}}^{-1} d\left(a, x_{3}, \ldots, x_{k}\right) .
\end{aligned}
$$

where $d\left(x_{2}, x_{3}\right)$ is certain measure on $\operatorname{Tr} x_{1}+\operatorname{Tr} x_{2}=1$ invariant under the translations by $\operatorname{Tr} x_{1}+$ $\operatorname{Tr} x_{2}=0$ and $d\left(a, x_{3}, \ldots, x_{k}\right)$ is certain measure on $a+\operatorname{Tr} x_{3}+\cdots+\operatorname{Tr} x_{k}=1$ invariant under the translations by $a+\operatorname{Tr} x_{3}+\cdots+\operatorname{Tr} x_{k}=0$. Both integrals on the right converge by induction assumption. Finally, we have

$$
\begin{aligned}
& \Gamma(c) \int_{S} c_{1}\left(x_{1}\right)\left|x_{1}\right|_{E_{1}}^{-1} \cdots c_{k}\left(x_{k}\right)\left|x_{k}\right|_{E_{k}}^{-1} d s \\
= & \int_{F} \psi(a) c(a)|a|^{-1} d a \int_{S} c_{1}\left(x_{1}\right)\left|x_{1}\right|_{E_{1}}^{-1} \cdots c_{k}\left(x_{k}\right)\left|x_{k}\right|_{E_{k}}^{-1} d s \\
= & \int_{F \times S} \psi\left(\operatorname{Tr}\left(a x_{1}+\cdots+a x_{k}\right)\right) c_{1}\left(a x_{1}\right)\left|a x_{1}\right|_{E_{1}}^{-1} \cdots c_{k}\left(a x_{k}\right)\left|a x_{k}\right|_{E_{k}}^{-1}|a|^{d-1} d s d a \\
= & \int_{E_{1} \times \cdots \times E_{n}} \psi\left(\operatorname{Tr}\left(y_{1}\right)+\cdots+\operatorname{Tr}\left(y_{k}\right)\right) c_{1}\left(y_{1}\right)\left|y_{1}\right|_{E_{1}}^{-1} \cdots c_{k}\left(y_{k}\right)\left|y_{k}\right|_{E_{k}}^{-1} \prod d y_{i} \\
= & \prod_{i=1}^{k} \Gamma_{E_{i}}\left(c_{i}\right) .
\end{aligned}
$$


We remark that if the map $\phi$ in Lemma 2.2 is replaced by $\phi\left(x_{1}, \ldots, x_{k}\right)=\sum_{i=1}^{k} \operatorname{Tr}_{i}\left(a_{i} x_{i}\right)$ for $a_{i} \in E_{i}^{*}$, the integral (2.8) is convergent under the same conditions on re $c_{i}$ 's and the result is (2.9) times $\prod_{i=1}^{k} c_{i}\left(a_{i}^{-1}\right)$. This can be proved using Lemma 2.2 and the change of variable $a_{i} x_{i} \mapsto x_{i}$.

For any $g \in F[x]$, let $n=\operatorname{deg} g$, and denote $F[x] /(g(x))$ by $F_{g}$ and equal $F_{g}$ with $F^{n}$ via the map

$$
\eta^{\prime}: F_{g} \rightarrow F^{n}, f(x)=b_{n-1} x^{n-1}+b_{n-2} x^{n-2}+\cdots+b_{0} \mapsto\left(b_{n-1}, b_{n-2}, \cdots, b_{0}\right) .
$$

Let $d_{g} f$ be the measure inherits from the product haar measure of $F^{n}$.

Let $G(x)$ be a monic separable polynomial over $F$. Assume $G(x)=\prod_{i=1}^{k} g_{i}(x)$, with $g_{i}(x)$ different monic irreducible polynomials over $F$. Then we have an isomorphism: $\varphi: E=F[x] /(G(x)) \rightarrow$ $\prod_{i=1}^{k} F[x] /\left(g_{i}(x)\right)$, such that

$$
\varphi(f)=\left(\varphi_{1}(f), \varphi_{2}(f), \cdots, \varphi_{k}(f)\right)=\left(f \bmod g_{1}, f \bmod g_{2}, \cdots, f \bmod g_{k}\right) .
$$

Let $g_{i}(x)=\prod_{j=1}^{d_{i}}\left(x-\alpha_{i j}\right)$ with $\alpha_{i j} \in \bar{F}, d_{i}=\operatorname{deg} g_{i}, 1 \leq i \leq k$. Define the Trace and Norm maps on $F_{g_{i}}$,

$$
\operatorname{Tr}_{g_{i}}(f):=\sum_{j=1}^{d_{i}} f\left(\alpha_{i j}\right) \text { and } \quad \mathrm{N}_{g_{i}}(f):=\prod_{j=1}^{d_{i}} f\left(\alpha_{i j}\right) .
$$

They are just usual trace and norm for field extension $F_{g_{i}}$ over $F$. Let $\psi_{i}: F_{g_{i}} \rightarrow S^{1}$ be the additive character $\psi_{i}(f)=\psi\left(\operatorname{Tr}_{g_{i}} f\right)$, it defines the Fourier transform on $\mathcal{S}\left(F_{g_{i}}\right)$ as $\mathcal{F} h(y):=$ $\int_{F_{g_{i}}} h(x) \psi_{i}(x y) d_{i} x$, where $d_{i} x$ is the unique measure on $F_{g_{i}}$ such that $\mathcal{F}$ is an isometry. We need to know the relations of Haar measures $d_{i} x$ and $d_{g_{i}} x$. For this purpose, we prove

Lemma 2.3. Let $D$ be a $n \times n$ non-degenerate symmetric matrix over $F$, and $d_{D} x$ be the unique Haar measure on $F^{n}$ such that the Fourier transform

$$
\mathcal{F}_{D} f(y)=\int_{F^{n}} f(x) \psi\left(x^{T} D y\right) d_{D} x
$$

is an isometry. Then $d_{D} x=|\operatorname{det} D|_{F}^{\frac{1}{2}} d x$, where $d x=d x_{1} \cdots d x_{n}$ is the product measure of the self-dual measure on $F$ determined by $\psi$.

Proof. By the uniqueness of the Haar measure, we have $d_{D} x=C d x$ for some positive scalar $C$. We first prove the case $F$ is non-Archimedean. Let $R$ be the ring of integers in $F, \pi \in R$ be a local parameter, $q=|R / \pi R|$. There exists $\delta \in \mathbb{Z}$ such that $\psi\left(\pi^{-\delta} R\right)=1$ and $\psi\left(\pi^{-\delta-1} R\right) \neq 1$. Then $\mathcal{F} 1_{R}(x)=q^{-\frac{\delta}{2}} 1_{\pi^{-\delta} R}(y)$, where $1_{S}$ denotes the characteristic function of set $S, \mathcal{F}$ is as in (1.2). It is easy to see that

$$
\mathcal{F}_{D} 1_{R^{n}}(x)=C q^{-\frac{n \delta}{2}} 1_{\pi^{-\delta} D^{-1} R^{n}}(x)
$$

The condition that that $\mathcal{F}_{D}$ is an isometry implies that $C=|\operatorname{det} D|_{F}^{\frac{1}{2}}$. For $F=\mathbb{R}$ or $\mathbb{C}$ we use Gaussian functions instead of $1_{R^{n}}$ to get the result.

From the Lemma, it's easy to see that $d_{i} x=\left|\Delta\left(g_{i}\right)\right|_{F}^{\frac{1}{2}} d_{g_{i}} x$. For any quasi-character $\chi$ on $F^{*}$, $\chi \circ \mathrm{N}$ is a quasi-character of $F_{g_{i}}^{*}$, we write $\Gamma_{E}(\chi \circ \mathrm{N})=\Gamma_{g_{i}}(\chi)$. The $F_{g_{i}}$-version of $(2.3)$ reads as

$$
\Gamma_{g_{i}}(\chi)=\int_{F_{g_{i}}} \psi\left(\operatorname{Tr}_{g_{i}}(x)\right) \chi\left(\mathrm{N}_{g_{i}}(x)\right)|x|_{F_{g_{i}}}^{-1} d_{i} x
$$

where $|x|_{F_{g_{i}}}$ is the absolute value on the field $F_{g_{i}}$ and we have $|x|_{F_{g_{i}}}=\left|\mathrm{N}_{g_{i}}(x)\right|_{F}$. 
Similarlly, define Trace and Norm map on $F_{G}$ as

$$
\operatorname{Tr}_{G}(f):=\sum_{i=1}^{k} \operatorname{Tr}_{g_{i}}\left(\varphi_{i}(f)\right) \text { and } \mathrm{N}_{G}(f):=\prod_{i=1}^{k} \mathrm{~N}_{g_{i}}\left(\varphi_{i}(f)\right) .
$$

Let $\psi: F_{G} \rightarrow S^{1}$ be the additive character $\phi(f)=\psi\left(\operatorname{Tr}_{G} f\right)$, it defines the Fourier transform on $\mathcal{S}\left(F_{G}\right)$ as $\mathcal{F}_{G} h(y):=\int_{F_{G}} h(x) \phi(x y) d_{*} x$, where $d_{*} x$ is the Haar measure such that $\mathcal{F}_{G}$ is an isometry. Using Lemma 2.3, we can prove that

$$
d_{*} x=|\Delta(G)|_{F}^{\frac{1}{2}} d_{G} x .
$$

It is also easy to see that $d_{*} x=\prod_{i=1}^{k} d_{i} \varphi_{i}(x)$.

For $f(x), g(x) \in F[x]$, assume $f(x)=a \prod_{i=1}^{n}\left(x-\alpha_{i}\right)$ and $g(x)=b \prod_{j=1}^{m}\left(x-\beta_{j}\right)$, where $a, b \in F$ and $\alpha_{i}, \beta_{j} \in \bar{F}, 1 \leq i \leq n, 1 \leq j \leq m$, the resultant of $f$ and $g$ is defined as

$$
R(f, g):=a^{m} b^{n} \prod_{i=1}^{n} \prod_{j=1}^{m}\left(\alpha_{i}-\beta_{j}\right)=a^{m} \prod_{i=1}^{n} g\left(\alpha_{i}\right)=(-1)^{m n} b^{n} \prod_{j=1}^{m} f\left(\beta_{j}\right) .
$$

In particular, for $g=b \in F^{*}$, we have $R(f, b)=b^{\operatorname{deg} f}$.

We will use the following properties of resultant, which can be proved by definition:

$$
\begin{aligned}
R\left(f, f^{\prime}\right) & =(-1)^{\frac{1}{2} n(n-1)} \Delta(f) ; \\
R\left(f, g_{1} g_{2}\right) & =R\left(f, g_{1}\right) R\left(f, g_{2}\right) ; \\
R\left(f_{1} f_{2}, g\right) & =R\left(f_{1}, g\right) R\left(f_{2}, g\right) .
\end{aligned}
$$

Then we have the following two propositions:

Proposition 2.4. For any $G(x) \in F[x]$ such that $G(x)=\prod_{i=1}^{k} g_{i}(x)$, with $g_{i}(x)$ different monic irreducible polynomial over $F$ such that $g_{i}(x)=\prod_{j=1}^{d_{i}}\left(x-\alpha_{i j}\right)$ with $\alpha_{i j} \in \bar{F}, d_{i}=\operatorname{deg} g_{i}, 1 \leq i \leq k$ and $n=\sum_{i=1}^{k} d_{i}$. Then we have

$$
\int_{f \in M_{n-1}} \chi \chi_{0}^{-1}(R(G, f)) d f=\chi_{0}(\Delta(G))^{-\frac{1}{2}} \chi\left(R\left(G, G^{\prime}\right)\right) \frac{\prod_{i=1}^{k} \Gamma_{g_{i}}(\chi)}{\Gamma\left(\chi^{n}\right)} .
$$

where $\chi_{0}(x)=|x|_{F}$, and $\chi$ is a quasi-character of $F^{*}$ such that $0<n$ re $\chi<1$.

Proof. View $M_{n-1}$ as a subset of $F_{G}$. For any $f \in F_{G}$, we have

$$
f(x)=\sum_{i=1}^{k} \sum_{j=1}^{d_{i}} f\left(\alpha_{i j}\right) \frac{\prod_{(k, l) \neq(i, j)}\left(x-\alpha_{k l}\right)}{\prod_{(k, l) \neq(i, j)}\left(\alpha_{i j}-\alpha_{k l}\right)}
$$

by Lagrange interpolation formula. Hence

$$
f \in M_{n-1} \Longleftrightarrow \sum_{i=1}^{k} \sum_{j=1}^{d_{i}} \frac{f\left(\alpha_{i j}\right)}{G^{\prime}\left(\alpha_{i j}\right)}=\sum_{i=1}^{k} \operatorname{Tr}_{g_{i}}\left(\frac{\varphi_{i}(f)}{\varphi_{i}\left(G^{\prime}\right)}\right)=1 .
$$

Let $\phi(g)=\sum_{i=1}^{k} \operatorname{Tr}_{g_{i}}\left(\varphi_{i}(g) / \varphi_{i}\left(G^{\prime}\right)\right)$, then $M_{n-1}=\phi^{-1}(1)$. And we have

$$
\int_{f \in M_{n-1}} \chi \chi_{0}^{-1}(R(G, f)) d f=\int_{f \in M_{n-1}} \prod_{i=1}^{k} \chi\left(\mathrm{N}_{g_{i}} \varphi_{i}(f)\right)\left|\varphi_{i}(f)\right|_{F_{g_{i}}}^{-1} d f .
$$


By Lemma 2.2 and the remark after its proof, we see the integral converges in the region $0<$ $n$ re $\chi<1$. At this point, we may change the variable $\varphi_{i}(f) / \varphi_{i}\left(G^{\prime}\right) \mapsto x_{i}$ and use Lemma 2.2 to prove (2.26), but it is messy to compute the change of various Haar measures, we choose to proceed directly as follows. We have an one-to-one map $\delta: F \times M_{n-1} \rightarrow F^{n}$, such that for $a \in F$, $f(x)=x^{n-1}+b_{n-2} x^{n-2}+\cdots+b_{0} \in M_{n-1}, \delta(a, f)=h=a f(x)=a x^{n-1}+a b_{n-2} x^{n-2}+\cdots+a b_{0}=$ $\left(a, a b_{n-2}, \cdots, a b_{0}\right)$. The Jacobian of $\delta$ is equal to $a^{n-1}$. Note that $F^{n} \backslash \delta\left(M_{n-1} \times F\right)$ has measure zero in $F^{n}$. So we have

$$
\begin{aligned}
& \Gamma\left(\chi^{n}\right) \int_{f \in M_{n-1}} \chi \chi_{0}^{-1}(R(G, f)) d f \\
= & \int_{F} \psi(a) \chi(R(G, a))|a|_{F}^{-1} d a \int_{f \in M_{n-1}} \chi \chi_{0}^{-1}(R(G, f)) d f \\
= & \int_{F_{G}} \psi(a) \chi \chi_{0}^{-1}(R(G, h)) d_{G} h \quad(\text { where } a \text { is the highest coefficience of the polynomial } h .) \\
= & \chi_{0}(\Delta(G))^{-\frac{1}{2}} \int_{F_{G}} \psi(a) \chi \chi_{0}^{-1}(R(G, h)) d_{*} h \\
= & \chi_{0}(\Delta(G))^{-\frac{1}{2}} \int_{\prod_{i=1}^{k} F_{g_{i}}} \psi\left(\sum_{i=1}^{k} \sum_{j=1}^{d_{i}} \frac{h\left(\alpha_{i j}\right)}{G^{\prime}\left(\alpha_{i j}\right)}\right) \chi_{0}^{-1}\left(\prod_{i=1}^{k} \prod_{j=1}^{d_{i}} h\left(\alpha_{i j}\right)\right) \prod_{i=1}^{k} d_{i} \varphi_{i}(h) \\
= & \chi_{0}(\Delta(G))^{-\frac{1}{2}} \chi \chi_{0}^{-1}\left(\prod_{i=1}^{k} \prod_{j=1}^{d_{i}} G^{\prime}\left(\alpha_{i j}\right)\right) \int_{\prod_{i=1}^{k}}^{k} \prod_{g_{i}} \prod_{i=1}^{k} \psi\left(\operatorname{Tr}_{g_{i}} \frac{\varphi_{i}(h)}{\varphi_{i}\left(G^{\prime}\right)}\right) \prod_{i=1}^{k} \chi \chi_{0}^{-1}\left(\mathrm{~N}_{g_{i}} \frac{\varphi_{i}(h)}{\varphi_{i}\left(G^{\prime}\right)}\right) \prod_{i=1}^{k} d_{i} \varphi_{i}(h) \\
= & \chi_{0}(\Delta(G))^{-\frac{1}{2}} \chi \chi_{0}^{-1}\left(R\left(G, G^{\prime}\right)\right) \prod_{i=1}^{k}\left|\varphi_{i}\left(G^{\prime}\right)\right|_{F_{g_{i}}} \prod_{i=1}^{k} \Gamma_{g_{i}}(\chi) \\
= & \chi_{0}(\Delta(G))^{-\frac{1}{2}} \chi \chi_{0}^{-1}\left(R\left(G, G^{\prime}\right)\right)\left|\prod_{i=1}^{k} \prod_{j=1}^{d_{i}} G^{\prime}\left(\alpha_{i j}\right)\right| \prod_{i=1}^{k} \Gamma_{g_{i}}(\chi) \\
= & \chi_{0}(\Delta(G))^{-\frac{1}{2}} \chi\left(R\left(G, G^{\prime}\right)\right) \prod_{i=1}^{k} \Gamma_{g_{i}}(\chi) \\
= & \chi_{0}(\Delta(G))^{\frac{1}{2}} \chi \chi_{0}^{-1}\left(R\left(G, G^{\prime}\right)\right) \prod_{i=1}^{k} \Gamma_{g_{i}}(\chi) \\
&
\end{aligned}
$$

where we used the relation $d_{G} h=\chi_{0}(\Delta(G))^{-\frac{1}{2}} d_{*} h$ in the third equation, which is just (2.23), a corollary of Lemma 2.3; and we used Lagrange interpolation in the fourth equation:

$$
h(x)=\sum_{i=1}^{k} \sum_{j=1}^{d_{i}} h\left(\alpha_{i j}\right) \frac{\prod_{(k, l) \neq(i, j)}\left(x-\alpha_{k l}\right)}{\prod_{(k, l) \neq(i, j)}\left(\alpha_{i j}-\alpha_{k l}\right)} \Rightarrow a=\sum_{i=1}^{k} \sum_{j=1}^{d_{i}} \frac{h\left(\alpha_{i j}\right)}{G^{\prime}\left(\alpha_{i j}\right)}
$$

and made change of variables in the sixth equation:

$$
\varphi_{i}(h) \mapsto \frac{\varphi_{i}(h)}{\varphi_{i}\left(G^{\prime}\right)}, \quad 1 \leq i \leq k .
$$


Proposition 2.5. For any $G(x) \in F[x]$ such that $G(x)=\prod_{i=1}^{k} g_{i}(x)$, with $g_{i}(x)$ different monic irreducible polynomial over $F$ such that $g_{i}(x)=\prod_{j=1}^{d_{i}}\left(x-\alpha_{i j}\right)$ with $\alpha_{i j} \in \bar{F}, d_{i}=\operatorname{deg} g_{i}, 1 \leq i \leq k$ and $\sum_{i=1}^{k} d_{i}=n-1$. Assume $G(0) G(1) \neq 0$, let $S=x(x-1) G$ and let $\alpha, \beta, \gamma$ be quasi-characters of $F^{*}$ such that $\operatorname{re} \alpha>0$, re $\beta>0$, re $\gamma>0$ and $\operatorname{re} \alpha+\operatorname{re} \beta+(n-1) \operatorname{re} \gamma<1$, then we have:

$$
\begin{aligned}
& \int_{f \in M_{n}} \alpha \chi_{0}^{-1}(f(0)) \beta \chi_{0}^{-1}(f(1)) \gamma \chi_{0}^{-1}(R(G, f)) d f \\
& =\alpha(-1) \chi_{0}(\Delta(G))^{-\frac{1}{2}} \alpha \gamma \chi_{0}^{-1}(G(0)) \beta \gamma \chi_{0}^{-1}(G(1)) \gamma\left(R\left(G, G^{\prime}\right)\right) \frac{\Gamma(\alpha) \Gamma(\beta) \prod_{i=1}^{k} \Gamma_{g_{i}}(\gamma)}{\Gamma\left(\alpha \beta \gamma^{n-1}\right)} .
\end{aligned}
$$

Proof. The proof is similar. View $M_{n}$ as a subset of $F_{S}$. For any $f \in F_{S}$, we have

$$
f(x)=\sum_{i=1}^{k} \sum_{j=1}^{d_{i}} f\left(\alpha_{i j}\right) \frac{x(x-1) \prod_{(k, l) \neq(i, j)}\left(x-\alpha_{k l}\right)}{\alpha_{i j}\left(\alpha_{i j}-1\right) \prod_{(k, l) \neq(i, j)}\left(\alpha_{i j}-\alpha_{k l}\right)}+f(0) \frac{(x-1) G(x)}{S^{\prime}(0)}+f(1) \frac{x G(x)}{S^{\prime}(1)} .
$$

by Lagrange interpolation formula. Hence

$$
f \in M_{n} \Longleftrightarrow \sum_{i=1}^{k} \sum_{j=1}^{d_{i}} \frac{f\left(\alpha_{i j}\right)}{S^{\prime}\left(\alpha_{i j}\right)}+\frac{f(0)}{S^{\prime}(0)}+\frac{f(1)}{S^{\prime}(1)}=\sum_{i=1}^{k} \operatorname{Tr}_{g_{i}}\left(\frac{\varphi_{i}(f)}{\varphi_{i}\left(S^{\prime}\right)}\right)+\frac{f(0)}{S^{\prime}(0)}+\frac{f(1)}{S^{\prime}(1)}=1 .
$$

Let $\phi(f)=\sum_{i=1}^{k} \operatorname{Tr}_{g_{i}}\left(\varphi_{i}(f) / \varphi_{i}\left(S^{\prime}\right)\right)+f(0) / S^{\prime}(0)+f(1) / S^{\prime}(1)$, then $M_{n}=\phi^{-1}(1)$. And we have

$$
\begin{aligned}
& \int_{f \in M_{n}} \alpha \chi_{0}^{-1}(f(0)) \beta \chi_{0}^{-1}(f(1)) \gamma \chi_{0}^{-1}(R(G, f)) d f \\
= & \int_{f \in M_{n}} \alpha(f(0))|f(0)|_{F}^{-1} \beta(f(1))|f(1)|_{F}^{-1} \prod_{i=1}^{k} \chi\left(\mathrm{N}_{g_{i}} \varphi_{i}(f)\right)\left|\varphi_{i}(f)\right|_{F_{g_{i}}}^{-1} d f
\end{aligned}
$$

By Lemma 2.2 and the remark after its proof, we see the integral converges in the region: re $\alpha$, re $\beta$, re $\gamma>$ 0 ; re $\alpha+\operatorname{re} \beta+(n-1)$ re $\gamma<1$. Similar to Proposition 2.4, we compute the integral as follows:

$$
\begin{aligned}
& \Gamma\left(\alpha \beta \gamma^{n-1}\right) \int_{f \in M_{n}} \alpha \chi_{0}^{-1}(f(0)) \beta \chi_{0}^{-1}(f(1)) \gamma \chi_{0}^{-1}(R(G, f)) d f \\
= & \int_{F} \psi(a) \alpha \beta(a) \gamma(R(G, a))|a|_{F}^{-1} d a \int_{f \in M_{n}} \alpha \chi_{0}^{-1}(f(0)) \beta \chi_{0}^{-1}(f(1)) \gamma \chi_{0}^{-1}(R(G, f)) d f \\
= & \int_{M_{n} \times F} \psi(a) \alpha \chi_{0}^{-1}(a f(0)) \beta \chi_{0}^{-1}(a f(1)) \gamma \chi_{0}^{-1}(R(G, a f))|a|_{F}^{n} d f d a \\
= & \int_{F_{S}} \psi(a) \alpha \gamma^{-1}(h(0)) \beta \gamma^{-1}(h(1)) \gamma \chi_{0}^{-1}(R(S, h)) d_{S} h(\text { where } a \text { is the highest coefficience of } h) \\
= & \chi_{0}(\Delta(S))^{-\frac{1}{2}} \int_{F_{S}} \psi(a) \alpha \gamma^{-1}(h(0)) \beta \gamma^{-1}(h(1)) \gamma \chi_{0}^{-1}(R(S, h)) d_{*} h(\text { Lemma } 2.3) \\
= & \chi_{0}(\Delta(S))^{-\frac{1}{2}} \int_{F_{S}} \psi\left(\sum_{i=1}^{k} \sum_{j=1}^{d_{i}} \frac{h\left(\alpha_{i j}\right)}{\alpha_{i j}\left(\alpha_{i j}-1\right) G^{\prime}\left(\alpha_{i j}\right)}+\frac{h(0)}{-G(0)}+\frac{h(1)}{G(1)}\right) \\
& \times \alpha \gamma^{-1}(h(0)) \beta \gamma^{-1}(h(1)) \gamma \chi_{0}^{-1}\left(h(0) h(1) \prod_{i=1}^{k} \prod_{j=1}^{d_{i}} h\left(\alpha_{i j}\right)\right) d_{*} h .
\end{aligned}
$$


Here we are using the Lagrange interpolation in the last equality to get the expression of $a$ in terms of $h$. Now via the isomorphism:

$$
F_{S} \cong F \times F \times F_{G}, h \mapsto(h(0), h(1), h \bmod G), d_{*} h \mapsto d_{*} h(0) \cdot d_{*} h(1) \cdot d_{* G} h .
$$

where $d_{* G} h$ is the haar measure on $F_{G}$ such that $\mathcal{F}_{G}$ is an isometry, we have

$$
\begin{aligned}
& \int_{F_{S}} \psi(a) \alpha \gamma^{-1}(h(0)) \beta \gamma^{-1}(h(1)) \gamma \chi_{0}^{-1}(R(S, h)) d_{*} h \\
= & \int_{F_{G}} \psi\left(\sum_{i=1}^{k} \sum_{j=1}^{d_{i}} \frac{h\left(\alpha_{i j}\right)}{\alpha_{i j}\left(\alpha_{i j}-1\right) G^{\prime}\left(\alpha_{i j}\right)}\right) \gamma \chi_{0}^{-1}\left(\prod_{i=1}^{k} \prod_{j=1}^{d_{i}} h\left(\alpha_{i j}\right)\right) d_{* G} h \\
& \times \int_{F} \psi\left(\frac{h(0)}{-G(0)}\right) \alpha \chi_{0}^{-1}(h(0)) d_{*} h(0) \int_{F} \psi\left(\frac{h(1)}{G(1)}\right) \beta \chi_{0}^{-1}(h(1)) d_{*} h(1) \\
= & \int_{\prod_{i=1}^{k} F_{g_{i}}} \prod_{i=1}^{k} \psi\left(\operatorname{Tr}_{g_{i}} \frac{\varphi_{i}(h)}{\varphi_{i}\left(x(x-1) G^{\prime}\right)}\right) \prod_{i=1}^{k} \gamma \chi_{0}^{-1}\left(\mathrm{~N}_{g_{i}}\left(\varphi_{i}(h)\right) \prod_{i=1}^{k} d_{i} \varphi_{i}(h)\right. \\
& \times \int_{F} \psi\left(\frac{h(0)}{-G(0)}\right) \alpha \chi_{0}^{-1}(h(0)) d_{*} h(0) \int_{F} \psi\left(\frac{h(1)}{G(1)}\right) \beta \chi_{0}^{-1}(h(1)) d_{*} h(1)
\end{aligned}
$$

where $\varphi_{i}\left(x(x-1) G^{\prime}\right)$ should be interpreted as $\varphi_{i}\left(x(x-1) G^{\prime} \bmod G\right)$. Now make several change of variables:

$$
\begin{aligned}
& \varphi_{i}(h) \mapsto \varphi_{i}(h) / \varphi_{i}\left(x(x-1) G^{\prime}\right), 1 \leq i \leq k ; \\
& h(0) \mapsto-h(0) / G(0) ; \\
& h(1) \mapsto h(1) / G(1) .
\end{aligned}
$$

We get:

$$
\begin{aligned}
& \Gamma\left(\alpha \beta \gamma^{n-1}\right) \int_{f \in M_{n}} \alpha \chi_{0}^{-1}(f(0)) \beta \chi_{0}^{-1}(f(1)) \gamma \chi_{0}^{-1}(R(G, f)) d f \\
= & \chi_{0}(\Delta(S))^{-\frac{1}{2}} \prod_{i=1}^{k} \gamma \chi_{0}^{-1}\left(\mathrm{~N}_{g_{i}}\left(\varphi_{i}\left(x(x-1) G^{\prime}\right)\right)\right)\left|\varphi_{i}\left(x(x-1) G^{\prime}\right)\right|_{F_{g_{i}}} \prod_{i=1}^{k} \Gamma_{g_{i}}(\gamma) \\
& \times \alpha \chi_{0}^{-1}(-G(0))|G(0)|_{F} \beta \chi_{0}^{-1}(G(1))|G(1)|_{F} \Gamma(\alpha) \Gamma(\beta) \\
= & \chi_{0}(\Delta(S))^{-\frac{1}{2}} \gamma \chi_{0}^{-1}\left(\prod_{i=1}^{k} \prod_{j=1}^{d_{i}} \alpha_{i j}\left(\alpha_{i j}-1\right) G^{\prime}\left(\alpha_{i j}\right)\right)\left|\prod_{i=1}^{k} \prod_{j=1}^{d_{i}} \alpha_{i j}\left(\alpha_{i j}-1\right) G^{\prime}\left(\alpha_{i j}\right)\right| \prod_{F}^{k} \Gamma_{g_{i}}(\gamma) \\
& \times \alpha(-G(0)) \beta(G(1)) \Gamma(\alpha) \Gamma(\beta) \\
= & \chi_{0}\left(G(0)^{2} G(1)^{2} \Delta(G)\right)^{-\frac{1}{2}} \gamma \chi_{0}^{-1}\left(G(0) G(1) R\left(G, G^{\prime}\right)\right)\left|G(0) G(1) R\left(G, G^{\prime}\right)\right|_{F} \prod_{i=1}^{k} \Gamma_{g_{i}}(\gamma) \\
& \times \alpha(-G(0)) \beta(G(1)) \Gamma(\alpha) \Gamma(\beta) \\
= & \alpha(-1) \chi_{0}(\Delta(G))^{-\frac{1}{2}} \alpha \gamma \chi_{0}^{-1}(G(0)) \beta \gamma \chi_{0}^{-1}(G(1)) \gamma\left(R\left(G, G^{\prime}\right)\right) \Gamma(\alpha) \Gamma(\beta) \prod_{i=1}^{k} \Gamma_{g_{i}}(\gamma) .
\end{aligned}
$$

which completes the proof of the proposition. 


\section{Proof of Theorem 1.1}

Proof. The proof is by induction. When $n=1$, the formula (1.8) is just (2.4). Now assume $S_{n-1}(\alpha, \beta, \gamma)$ converges for any $\alpha, \beta, \gamma$ in the region $R_{n-1}$ and we have

$$
S_{n-1}(\alpha, \beta, \gamma)=\prod_{j=0}^{n-2} \frac{\Gamma\left(\alpha \gamma^{j}\right) \Gamma\left(\beta \gamma^{j}\right) \Gamma\left(\gamma^{j+1}\right)}{\Gamma\left(\alpha \beta \gamma^{n+j-1}\right) \Gamma(\gamma)}
$$

Consider the double integral:

$$
T_{n}:=\int_{P \in M_{n-1}} \int_{Q \in M_{n}} \alpha \chi_{0}^{-1}(Q(0)) \beta \chi_{0}^{-1}(Q(1)) \gamma \chi_{0}^{-1}(R(P, Q)) d Q d P .
$$

By Propostion 2.5, we get

$$
\begin{aligned}
& T_{n, P} \\
:= & \int_{P \in M_{n-1}}\left(\int_{Q \in M_{n}} \alpha \chi_{0}^{-1}(Q(0)) \beta \chi_{0}^{-1}(Q(1)) \gamma \chi_{0}^{-1}(R(P, Q)) d Q\right) d P \\
= & \int_{P \in M_{n-1}} \alpha(-1) \chi_{0}^{-\frac{1}{2}}(\Delta(P)) \alpha \gamma \chi_{0}^{-1}(P(0)) \beta \gamma \chi_{0}^{-1}(P(1)) \gamma\left(R\left(P, P^{\prime}\right)\right) \frac{\Gamma(\alpha) \Gamma(\beta) \prod_{i=1}^{k} \Gamma_{g_{i}}(\gamma)}{\Gamma\left(\alpha \beta \gamma^{n-1}\right)} d P \\
= & \alpha^{n}(-1) S_{n-1}(\alpha \gamma, \beta \gamma, \gamma) \frac{\Gamma(\alpha) \Gamma(\beta) \Gamma(\gamma)^{n-1}}{\Gamma\left(\alpha \beta \gamma^{n-1}\right)}
\end{aligned}
$$

valid in the region

$$
\begin{aligned}
& \text { re } \alpha, \operatorname{re} \beta, \operatorname{re} \gamma>0 \\
& \text { re } \alpha+\operatorname{re} \beta+(n-1) \operatorname{re} \gamma<1 \\
& \operatorname{re} \alpha+1+\operatorname{re} \beta+1+2(n-2) \operatorname{re} \gamma<1
\end{aligned}
$$

which is exactly the region $R_{n}$. Note that the absolute value of the intergrand is the same as replacing $\alpha, \beta, \gamma$ with $\chi_{0}^{\text {re } \alpha}, \chi_{0}^{\text {re } \beta}, \chi_{0}^{\text {re } \gamma}$. Hence the we have $T_{n}=T_{n, P}=T_{n, Q}$ in $R_{n}$ by FubiniTonelli theorem, where $T_{n, Q}$ is defined by the following

$$
\begin{aligned}
& T_{n, Q} \\
:= & \int_{Q \in M_{n}}\left(\int_{P \in M_{n-1}} \alpha \chi_{0}^{-1}(Q(0)) \beta \chi_{0}^{-1}(Q(1)) \gamma \chi_{0}^{-1}(R(P, Q)) d P\right) d Q . \\
= & \int_{Q \in M_{n}} \alpha \chi_{0}^{-1}(Q(0)) \beta \chi_{0}^{-1}(Q(1)) \chi_{0}(\Delta(Q))^{-\frac{1}{2}} \gamma\left(R\left(Q, Q^{\prime}\right)\right) \frac{\prod_{i=1}^{k} \Gamma_{g_{i}}(\gamma)}{\Gamma\left(\gamma^{n}\right)} d Q \\
= & \alpha^{n}(-1) S_{n}(\alpha, \beta, \gamma) \frac{\Gamma(\gamma)^{n}}{\Gamma\left(\gamma^{n}\right)}
\end{aligned}
$$

here we are using Proposition 2.4 in the second equation. Thus $S_{n}(\alpha, \beta, \gamma)$ converges in the region $R_{n}$, and $T_{n, P}=T_{n, Q}$ gives us

$$
S_{n}(\alpha, \beta, \gamma)=S_{n-1}(\alpha \gamma, \beta \gamma, \gamma) \frac{\Gamma(\alpha) \Gamma(\beta) \Gamma\left(\gamma^{n}\right)}{\Gamma\left(\alpha \beta \gamma^{n-1}\right) \Gamma(\gamma)}=\prod_{j=0}^{n-1} \frac{\Gamma\left(\alpha \gamma^{j}\right) \Gamma\left(\beta \gamma^{j}\right) \Gamma\left(\gamma^{j+1}\right)}{\Gamma\left(\alpha \beta \gamma^{n+j-1}\right) \Gamma(\gamma)}
$$

which completes the proof of the main theorem. 
As a special case, we consider when $F=\mathbb{C}$. We take $\psi(z)=\psi(x+i y)=e^{4 \pi i x}$ as in [Tate]. The self-dual Haar measure $d z$ is then twice the usual Lebesgue measure on $\mathbb{C}$. Note that $|z|_{\mathbb{C}}=|z|^{2}$. The map

$$
\Phi_{n}: \mathbb{C}^{n} \rightarrow M_{n}, \quad\left(z_{1}, \cdots, z_{n}\right) \mapsto f(z)=\prod_{i=1}^{n}\left(z-z_{i}\right)=z^{n}+b_{n-1} z^{n-1}+\cdots+b_{0}
$$

is surjective and generically $n$ ! to 1 . It is known that the Jacobian of this map is $\operatorname{Jac} \Phi_{n}\left(z_{1}, \cdots, z_{n}\right)=$ $\prod_{i<j}\left(z_{i}-z_{j}\right)[$ Ser]. Thus

$$
\left|\operatorname{Jac} \Phi_{n}\left(z_{1}, \cdots, z_{n}\right)\right|_{\mathbb{C}}=\left|\prod_{i<j}\left(z_{i}-z_{j}\right)\right|_{\mathbb{C}}=|\Delta(f)|_{\mathbb{C}}^{\frac{1}{2}} .
$$

So we have

$$
\begin{aligned}
S_{n}(\alpha, \beta, \gamma) & =\alpha^{n} \gamma^{\frac{n(n-1)}{2}}(-1) \int_{f \in M_{n}} \alpha \chi_{0}^{-1}(f(0)) \beta \chi_{0}^{-1}(f(1)) \gamma \chi_{0}^{-\frac{1}{2}}(\Delta(f)) d f \\
& =\frac{1}{n !} \int_{\mathbb{C}^{n}} \alpha \chi_{0}^{-1}\left(\prod_{i=1}^{n} z_{i}\right) \beta \chi_{0}^{-1}\left(\prod_{i=1}^{n}\left(1-z_{i}\right)\right) \gamma\left(\prod_{i \neq j}\left(z_{i}-z_{j}\right)\right) \prod_{i=0}^{n} d z_{i} .
\end{aligned}
$$

Then by Thm 1.1, we get:

$$
\int_{\mathbb{C}^{n}} \prod_{i=1}^{n} \alpha \chi_{0}^{-1}\left(z_{i}\right) \beta \chi_{0}^{-1}\left(1-z_{i}\right) \prod_{i \neq j} \gamma\left(\left(z_{i}-z_{j}\right)\right) \prod_{i=0}^{n} d z_{i}=n ! \prod_{j=0}^{n-1} \frac{\Gamma_{\mathbb{C}}\left(\alpha \gamma^{j}\right) \Gamma_{\mathbb{C}}\left(\beta \gamma^{j}\right) \Gamma_{\mathbb{C}}\left(\gamma^{j+1}\right)}{\Gamma_{\mathbb{C}}\left(\alpha \beta \gamma^{n+j-1}\right) \Gamma_{\mathbb{C}}(\gamma)}
$$

valid in the region $R_{n}$. If $\alpha, \beta, \gamma$ are all unramified, i.e. $\alpha=|\cdot|_{\mathbb{C}}^{a}, \beta=|\cdot|_{\mathbb{C}}^{b}, \gamma=|\cdot|_{\mathbb{C}}^{c}$, for some $a, b, c \in \mathbb{C}$, then formula (3.10) becomes:

$$
\begin{aligned}
& \int_{\mathbb{C}^{n}} \prod_{i=1}^{n}\left|z_{i}\right|^{2 a-2}\left|1-z_{i}\right|^{2 b-2} \prod_{1 \leq i<j \leq n}\left|z_{i}-z_{j}\right|^{4 c} \prod_{i=0}^{n} d z_{i} \\
= & n ! \prod_{j=0}^{n-1} \frac{\Gamma_{\mathbb{C}}(a+j c) \Gamma_{\mathbb{C}}(b+j c) \Gamma_{\mathbb{C}}((j+1) c)}{\Gamma_{\mathbb{C}}(a+b+(n+j-1) c) \Gamma_{\mathbb{C}}(c)} \\
= & \frac{\prod_{j=0}^{n-1} 2 \sin (\pi(a+j c)) \sin (\pi(b+j c)) \sin (\pi(j+1) c)}{n ! \prod_{j=0}^{n-1} \sin (\pi(a+b+(n+j-1) c)) \sin (\pi c)} S_{n}(a, b, c)^{2}
\end{aligned}
$$

where for any $s \in \mathbb{C}$ with $0<$ re $s<1$,

$$
\Gamma_{\mathbb{C}}(s):=\Gamma_{\mathbb{C}}\left(|\cdot|_{\mathbb{C}}^{s}\right)=\frac{(2 \pi)^{1-s} \Gamma(s)}{(2 \pi)^{s} \Gamma(1-s)}=2^{1-2 s} \pi^{-2 s} \Gamma(s)^{2} \sin (\pi s) .
$$

(cf. [Tate]) and $S_{n}(a, b, c)$ is defined in (1.1). The formula (3.11) is the same as the formula obtained by Aomoto [Ao].

\section{REFERENCES}

[AAR] G.E. Andrews, R. Askey, R. Roy, Special Functions, Cambridge Univ. Press, 1999.

[An] G.W. Anderson, The evaluation of Selberg sums, C. R. Acad. Sci. Paris Sér. I Math. 311 (1990), 469-472.

[An2] G.W. Anderson, A short proof of Selberg's generalized beta formula, Forum Math. 3 (1991), 415-417.

[Ao] K. Aomoto, On the complex selberg integral, Quart. J. Math. Oxford (2), 38 (1987), 385-399. 
[E1] R.J. Evans, Identities for products of Gauss sums over finite fields, L'Enseignement Math. 27 (1981), $197-209$.

[E2] R.J. Evans, The evaluation of Selberg character sums, L'Enseignement Math. 37 (1991), 235-248.

[FW] P.J. Forrester, S.O. Warnaar, The importance of the Selberg integral, Bull. Amer. Math. Soc. (N.S.) 45 (2008), 489-534.

[GGPS] I.M. Gelfand, M.I. Graev, and I.I. Piatetskii-Shapiro, Representation Theory and Automorphic Functions (Saunders, Philadelphia, PA, 1996).

[Sel] A. Selberg, Remarks on a multiple integral, Norsk. Mat. Tidsskr. 24 (1944), 71-78.

[Ser] J.-P. Serre, Une "formule de masse" pour les extensions totalement ramifiés de degré donné d'un corps local, C.R. Acad. Sc. Paris Série A 286 (1978), 1031-1036.

[Tate] J. Tate, Fourier analysis in number fields, and Hecke's zeta-functions, Princeton, May 1950, thesis; reproduced in Algebraic Number Theory (Proc. Instructional Conf., Brighton, 1965) pp. 305-347, Acad, Press 1967.

[Tai] M.H. Taibleson, Fourier Analysis on local fields, Princeton university press, 1975.

School of Mathematical Science, Zhejiang University, Hangzhou 310027, Zhejiang, P.R. China

E-mail address: zenanfu@zju.edu.cn

Department of Mathematics, The Hong Kong University of Science and Technology, Clear Water Bay, Kowloon, Hong Kong

E-mail address: mazhu@ust.hk 